\title{
SisTeMA PERIÓdico DE LOS ELEMENTOS QUÍMICOS, PROGRESO CONCEPTUAL Y DIDÁCTICA
}

\section{Periodic system of the chemical elements, CONCEPTUAL PROGRESS AND DIDACTICS}

\author{
María Victoria Alzate Cano* \\ vicky@matematicas.udea.edu.co
}

\section{Abstrac}

This article presents an approximation study to the intellectual progress, ocurred between the years 1790 to 1920 , of the periodic system of the chemical elements. It has been inspired by the Imre Lakatos' epistemological perspective. It analyse the roll of this study for the scholar formation on teachers and also in the planification of didactic activities oriented to promove a significative understandig of it system.

Keywords: Periodic System of the Chemical Elements; Epistemology of Imre Lakatos; Didactics

\section{RESUMEN}

Este artículo presenta un estudio aproximado del progreso intelectual del sistema periódico de los elementos químico en el período comprendido entre 1790 y 1920, desde la perspectiva de la epistemología de Imre Lakatos, y el papel que desempeña esta clase de estudios para la formación de docentes y el diseño de actividades didácticas orientadas a estimular una comprensión significative de este sistema.

Palabras Clave: sistema periódico de los elementos químicos, epistemología de Imre Lakatos, didáctica. 


\section{INTRODUCCIÓN}

El trabajo aquí presentado es la primera parte de un estudio sobre el sistema periódico de los elementos químicos, fundamentado en el progreso conceptual del criterio organizador del cambio del orden creciente de las masas atómicas y la periodicidad de las propiedades químicas, la valencia, al orden creciente del número atómico y la periodicidad de las propiedades químicas.

El estudio posee una doble intencionalidad: Primero, una reflexión acerca del progreso y refinamiento conceptual del sistema periódico de los elementos químicos, en términos de cambios progresivos y regresivos de series de teorías científicas y segundo, generar un reflexión acerca de la enseñanza y el aprendizaje de la ley de la periodicidad de los elementos químicos.

El abordaje de esta presentación inicia con una exposición breve de las ideas centrales de la epistemología "Metodología de los Programas de Investigación Científica" de Imre Lakatos; luego, con algunas razones que explican la importancia del progreso conceptual del sistema periódico de los elementos químicos para la enseñanza y el aprendizaje de la química; continúa con varios aspectos del crecimiento conceptual del sistema periódico a la luz del desarrollo de series de teorías, avanza con una descripción de la tabla periódica y concluye con algunas reflexiones e interrogantes sobre sus implicaciones didácticas.

Este estudio está fundamentado, además de la bibliografía citada a lo largo del texto, en las publicaciones de Seaborg (1985), Roundy (1989), Marc (1989), Gorin (1996), Scerri (1998), Scerri (2000) y Strathern (2000).

Esta producción hace parte del proyecto de investigación titulado "Sistema periódico en Química: qué y como enseñar para posibilitar un aprendizaje significativo en alumnos de primer nivel universitario", propuesta auspiciada por Colciencias y la U. de A.

\section{EPISTEMOLOGÍA de IMRE LAKATOS}

La nueva epistemología de la ciencia plantea el conocimiento como una construcción humana, la cual involucra la información disponible, los modelos teóricos, la formación y los valores sociales de los científicos; es una creación del espíritu humano que exige un amplio despliegue de la imaginación. Tal vez, lo más novedoso de la nueva filosofía de la ciencia, sean sus desarrollos por ex- plicar el progreso de la ciencia como un proceso evolutivo. Su interés, es el cambio de las teorías a formas cada vez más refinadas y explicativas del mundo, con mayor capacidad de predicción y con mayor contenido empírico que las predecesoras, en oposición al positivismo que pretendía verificar o justificar las teorías.

Imre Lakatos (1983), propone "La Metodología de los Programas de Investiga- 
ción Científica". Según esta metodología, los grandes descubrimientos científicos, los de mayor significatividad, son programas de investigación que pueden evaluarse con base en problemáticas progresivas y estancadas. Esta metodología fortalece el racionalismo y transforma las teorías en series de teorías o programas de investigación, e introduce la historia como elemento principal para evaluarlos.

Problemática progresiva se refiere a un programa de investigación cuyo progreso teórico anticipa su desarrollo empírico, esto es, capacidad teórica para predecir nuevos hechos con un alto grado de éxito. Un programa de investigación es estancado o regresivo, si su desarrollo teórico queda rezagado respecto a su desarrollo empírico, es decir, cuando invoca explicaciones a posteriori o predice hechos ya anticipados por otro programa. La idea de progreso científico está asociada a la confrontación entre series de teorías rivales o programas rivales y el desarrollo empírico de éstas. La metodología de programas de investigación científica adopta el criterio de preferencia por el mejor programa.

El mejor programa es aquel que tiene un exceso de contenido empírico y cuya teoría conduce al descubrimiento de hechos nuevos inesperados, posee el contenido empírico de su predecesor y es capaz de explicar situaciones contradichas por programas rivales. La experiencia no puede falsar una teoría, debe facilitar el surgimiento de una nueva teoría con mayor poder heurístico, esto es, una teoría solo puede ser reemplazada o archivada por otra teoría mejor, por una teoría que exceda en contenido empírico a sus predecesoras.

Todo programa de investigación científica se caracteriza por un núcleo firme, llamado heurística negativa y por un cinturón protector nombrado heurística positiva. La heurística negativa es el conjunto de los aspectos esenciales de la teoría, es decir, de los supuestos fundamentales considerados irrefutables, los cuales son leyes expresadas mediante el formalismo matemático y/o en forma de enunciados proposicionales. La heurística positiva esta formada por el conjunto de hipótesis auxiliares, susceptibles de verificación, refutación y cambio; es el espacio de las contrastaciones para defender el centro firme, es ajustada y reajustada y puede ser modificada en su totalidad.

En la heurística positiva los científicos construyen modelos, cada uno de mayor cualificación que el anterior, con mayor contenido teórico para explicar de un modo más adecuado el campo de investigación del programa.

Los programas de investigación son flexibles, tienen problemas y enigmas sin resolver y los científicos a posteriori aceptan uno u otro programa como progresivo.

\section{AsPeCtos ACERCA DE LA IMPORTANCIA DEL PROGRESO CONCEPTUAL DEL SISTEMA PERIÓDICO}

La clasificación periódica de los elementos químicos, es una construcción científica, acompañada de estudios empíricos y de series de teorías de la química y la física, los cuales han enriquecido los contenidos del sistema y en particular las relaciones de periodicidad de un gran número de propiedades químicas y físicas de diversa naturaleza. Es un sistema facilitador del análisis, la interpretación, la inferencia y la predicción del comportamiento químico y físico de los elementos, las sustancias simples y series homólogas de sustancias compuestas, así como de propie- 
dades espectroscópicas y nucleares, y de las características de la estructura molecular que representa a las sustancias simples y ampliado para clases de sustancias compuestas, así como para átomos y sus formas iónicas.

Para efectos de la enseñanza y el aprendizaje, es pertinente el conocimiento del progreso conceptual en espiral del sistema periódico, en particular, su estudio como programa de investigación con una heurística negativa centrada en el progreso empírico y racional de la metodología analítica del análisis químico, lo cual ha dado origen a una hipótesis atómica evolucionada de las masas atómicas y la valencia, al número atómico y la valencia; una heurística positiva construida con varios programas (Subprogramas de investigación) desde diferentes vertientes de la química y la física tanto empíricas como teóricas, la cual se constituye en un gran conjunto de metodologías analíticas y de series de teorías que progresan a la luz del refinamiento de los modelos moleculares tanto para moléculas mononucleares (Átomos) como para las polinucleares.

El conocimiento del progreso conceptual en espiral del sistema periódico es una vía pedagógica para la reflexión acerca del significado de creación científica como algo relativo, que evoluciona en el tiempo con la participación interdisciplinaria de grupos de científicos de diversas áreas del conocimiento, con la creación de modelos cada vez con mayor grado de racionalidad, con la evolución del concepto elemento químico vinculado de modo dialéctico a las técnicas de análisis químico y su crecimiento racional, así como a los desarrollos del modelo atómico. Además, es una opción para propiciar el desarrollo con- ceptual de los estudiantes, mediante el diseño de actividades dirigidas a realizar diversas clasificaciones de sustancias y establecer relaciones de periodicidad, orientadas con diversos criterios de ordenación fundamentados en la conceptualización molar, molecular y eléctrica de la química. Permite también la comprensión y la familiaridad con formatos diversos del sistema periódico y de los conceptos implicados en la gran cantidad de datos presentados, en la perspectiva de una visión amplia y flexible, abierta al diálogo sobre las diferencias y las semejanzas del ordenamiento de los elementos químicos y de las propiedades químicas y físicas de las sustancias y sus vínculos profundos con las metodologías de análisis químico y los modelos moleculares.

\section{Precursores de D. MendeléleV}

La «Búsqueda de los Elementos Químicos», título dado por Asimov (1993) a una de sus obras, es el nombre dado en este artículo al programa de investigación para identificar los constituyentes de los cuerpos del mundo material, el cual a la fecha tiene una vigencia de 2600 años.

La metodología de este análisis se basa en la idea lakatosiana de la reconstrucción histórica y la identificación del núcleo firme y la heurística positiva de los procesos de progreso científico

Este programa, orientado a identificar y clasificar los elementos químicos tiene un núcleo firme sintetizado en los siguientes puntos:

1. La pregunta formulada por Tales de Mileto en el ano 640 a.n.e.:

¿De qué materiales está hecho el sol, la luna, la tierra y los seres vivos sobre el planeta? (Asimov, 1990). 
Durante cerca de 2000 años, el cinturón de seguridad divagó acerca del concepto de elemento. A partir de 1661, el concepto operativo dado por el físico y químico inglés Robert Boyle (16271691) se convierte en parte del núcleo firme del programa sobre la búsqueda de los elementos químicos.

\section{La definición operacional de elemento} químico. Esta definición esta fundamentada en los trabajos de R. Boyle, quien tenía el objetivo de identificar los verdaderos elementos químicos e impugnar la teoría de los cuatros elementos aristotélicos: tierra, agua, aire y fuego, a fin de superar las ideas peripatéticas y paracelsianas acerca del concepto de elemento. A la pregunta ¿Como identificar los elementos?, R. Boyle da una respuesta operacional, lograrlos mediante métodos de combinación química de sustancias para formar nuevas sustancias compuestas y descomponer éstas en sus elementos, o por métodos de separación. Este camino histórico comienza cuando a mediados del siglo XVII, el químico R. Boyle en su obra «El Químico Escéptico», define como elemento:

«La muestra que no puede ser descompuesta o separada en otros cuerpos diferentes» (Moore, 1953).

Esta definición ha progresado y por ende modificado en su contenido operacional como metodología analítica de la química y en su contenido racional, en cuanto que alcanzar la sustancia elemental implica el refinamiento de las técnicas de análisis, mediciones indirectas y procedimientos formales para alcanzar los resultados.

Las dos cuestiones anteriores son consideradas como la heurística negativa del programa, el cual ha evolucionado en su cinturón de seguridad, al ser refinados los métodos de separación y las conjeturas y teorías acerca de los elementos químicos. La heurística positiva ha avanzado en varias etapas, las cuales han implicado en primer lugar, procesos de sistematización de datos empíricos y de clasificación de los elementos químicos.

Los datos empíricos, cualesquiera sea su origen, ya sea por la percepción directa o indirecta mediante diferentes medios instrumenta-les, no dejan de ser informaciones puntuales que poco dicen por si mismos. Para trascender este nivel, es fundamental el propósito de dar respuesta a problemas científicos. Esto requiere de la necesaria ordenación de los datos de modo claro y sistemático en función de los conocimientos buscados, lo cual exige delimitar un criterio de clasificación que surge desde el seno mismo de la organización de la información disponible. De este modo, el criterio de clasificar por semejanzas y diferencias de la similaridad química implica conocer los constituyentes más importantes de la organización, conocerlos en sus apariencias reales y en sus cualidades externas e internas.

Sabino (1999) plantea que toda ordenación debe someterse a una normas lógicas, partiendo de los atributos comunes y esenciales a todos los objetos 0 fenómenos coleccionados, de tal modo que las categorías más globales subsuman las más específicas hasta llegar a las más particulares. Organizar de un modo sistemático, según un criterio empírico o teórico y unas normas lógicas de generalización para construir estructuras clasificatorias de generalidades, no se desarrolla a ciegas y es esencial idear un panorama estructural de los diferentes objetos o eventos motivo de un estudio clasificatorio. 
La química incorporó desde muy temprano la metodología descriptiva y clasificatoria de las sustancias y sus comportamientos químicos y físicos. Para la penúltima década del siglo XVIII, era tal el acervo de conocimientos empíricos sobre las sustancias y sus modificaciones para entonces conocidas, que la metodología científica de descripción y clasificación, irrumpe para dar origen a una de las más grandes creaciones científicas, el sistema periódico de los elementos químicos.

Lavoisier (1982) publicó en 1797, una serie de tablas en las cuales lista grupos de sustancias y una investigación de sus propiedades. Entre estas tablas, se encuentra una titulada "tabla de las sustancias simples", en la cual lista treinta y tres (33) sustancias simples clasificadas en cuatro clases: simples pertenecientes a los tres reinos, simples metálicas, simples no metálicas y simples salificables. Los desarrollos posteriores corrigieron varias de las sustancias simples enunciadas y las ordenaron en tres grandes clases: metálicas, metaloides y no metálicas.

En esta clasificación unidimensio-nal, la cual recibió el apoyo de eminentes químicos como A. Foucroy (1755-1809), L. Berthollet (1748-1822) y L. Guyton (1737-1816), reside el núcleo de la química moderna para ordenar y describir elementos químicos. La dilucidación de los criterios de organización de los elementos químicos llegó con los intentos subsiguientes de clasificar elementos.

En 1817, J. Döbereiner (1780-1849) inició un orden sistemático de los elementos en tríadas, en el cual los agrupaba de modo unidimensional de acuerdo a la similaridad química, este orden era a la vez, un orden creciente de las masas atómicas de los elementos. Esta contribución es conocida como la ley de las tríadas: La masa atómica del elemento intermedio era aproximadamente el promedio de las masas de los elementos de los extremos. Esta primera idea de periodicidad de las tríadas fue elevada por P. Kramers a la categoría de orden bidimensional, al plantear que ciertos elementos, dada su similaridad química, podían pertenecer a dos tríadas perpendiculares entre sí, es decir, los elementos organizados en filas y columnas que se cruzan pueden ser comparados en dos direcciones. Esta idea, de la perpendicularidad de la similaridad química, es decir, un elemento es común a dos tríadas dispuestas de modo perpendicular, es la clave del desarrollo de los avances posteriores manifiestos en el trabajo de J. Newlands (1838-1898) en 1866, "Ley de las Octavas". En ésta, se expresa un orden creciente de las masas atómicas de los elementos en una fila y la similaridad química en las columnas, cada ocho elementos a partir de uno dado.

Otro trabajo, como el de A. E. Chancourtois (1820-1886) en 1862, ordenaba los elementos en orden creciente de sus masas atómicas en una organización de forma helicoidal, lo cual le hizo notar que aquellos que tenían propiedades químicas similares quedaban ubicados en la misma línea vertical. En 1870 , Lothar Meyer (1830-1895) publicó un trabajo basado en un estudio comparativo de los volúmenes atómicos y las temperaturas de fusión según un orden creciente de las masas atómicas.

Estos diferentes intentos de clasificación de los elementos y de creación de una ley de periodicidad, no tuvieron el éxito obtenido por D. Mendeléiev (18341907). ¿Cuáles son las razones de esta situación? 
El éxito de D. I. Mendeléiev, se debe no sólo a los hechos que también conocían los químicos contemporáneos de él, sino en primer lugar a que ya se había formado un concepto claro sobre los grupos de los elementos semejantes por sus propiedades químicas; de otra parte, tenía nueva información acerca de los Ilamados elementos raros; en tercer lugar, por las importantes correcciones planteadas, al parecer arbitrarias, de las magnitudes de las masas atómicas de varios elementos y finalmente, a la convicción de que no todos los elementos eran conocidos. Estas ideas le permitieron concebir espacios libres para los futuros descubrimientos. La fina intuición y su gran audacia científica para concebir la regularidad periódica, es lo que le permitió proceder a corregir información anterior y predecir nuevas características aún de elementos no conocidos.

Mientras Mendeléiev comparó las masas atómicas de todos los elementos y las enlazó de una forma lógica con las propiedades químicas, la valencia, sus predecesores sólo comparaban los elementos semejantes entre sí y no podían por lo tanto enlazar los grupos de elementos y establecer un orden cruzado de filas y columnas con alto poder de predicción. Estas ideas, están integradas al muy fuerte pensamiento filosófico de Mendeléiev expresado varias décadas después, Mendeléiev (1905, XIV), centrado en la noción clásica de una materia primaria, que dados los avances encontraría respuesta en las investigaciones relacionadas con los fenómenos ópticos y eléctricos y las teorías asociadas e éstos, materia primaria más allá de las sustancias perceptibles y sus respectivas características.

\section{EtAPA dE INVESTIGACIÓN CIENTíFICA De Dimitri l. MendeléieV}

La investigación de Mendeléiev está centrada en la hipótesis proutiana de que la masa atómica es un múltiplo entero de la masa del hidrógeno. Ésta hipótesis atómica apoyada en una serie de proposiciones observacionales y relacionadas con la similaridad de las propiedades químicas de los elementos, constituye la heurística positiva del programa. El cinturón protector provisto de una gran riqueza de datos empíricos y de una gran posibilidad de establecer con ellos relaciones de periodicidad, fue evidenciado por Mendeléiev cuando encontró que los volúmenes atómicos de las sustancias simples eran una función periódica de las masas atómicas, relación de periodicidad que encontró también válida para la valencia superior o valencia primaria de los elementos en la formación de hidruros y de óxidos.

La ley periódica es una ordenación expresada como un correlato de las masas atómicas en las filas y la similaridad de las propiedades químicas en las columnas, representada en un formato bidimensional que relaciona de modo periódico el orden creciente de las masa atómicas con la similaridad de las propiedades químicas o la valencia. A finales de 1870 Mendeléiev enuncia la ley de periodicidad como ley de la naturaleza bajo el título "Sistema Natural de los Elementos y su Aplicación para Definir las Propiedades de los Elementos no Descubiertos" o "Ley de la Periodicidad", y en 1871 la nombra "Sistema Periódico"; expresó su formulación en los siguientes términos: "las propiedades de los elementos y, por esta causa, también las propiedades de los cuerpos simples y de los compuestos que forman, se encuentran en relación periódica con su peso atómico". (Petriánov, Trífonov 1981).

Este sistema Periódico, no sólo contiene una sistematización de una hipótesis atómica y de los conocimientos adquiridos de lo que antes era una disper- 
sión de sustancias y sus propiedades, sino principalmente, por un lado, el replanteamiento no previsto por químicos experimentales, de las masas atómicas de elementos como el Torio, Itrio, Cerio, Uranio e Indio y, por otro, los cerca de treinta y seis espacios libres que planteó en el sistema. Pero lo más trascendental, que evidencia el gran aporte de Mendeléiev, fue que algunos de estos espacios libres, constituían la predicción de la existencia y de las propiedades de más de diez elementos no conocidos, entre ellos: Germanio, Escandio, Galio, Radio, Polonio, Actinio, Tecnecio, Renio y Hafnio. La tabla periódica aparece como una totalidad orgánica del conjunto de sustancias y propiedades en otro tiempo indeterminado.

El éxito de Medendeléiev se debió a la verificación de sus predicciones cuando posteriormente entre 1875 y 1890 se obtuvo en los laboratorios los elementos galio, escandio y germanio. Estos hallazgos eran coherentes con las predicciones de Mendeléiev, referidas no sólo a las propiedades y existencia de algunas de las correspondientes sustancias compuestas, sino también al surgimiento de nuevos métodos químicos para lograr su identificación, como es el análisis espectroscópico creado en el período 1860-1870 y refinado en años posteriores, lo cual permitió la identificación del elemento Galio y otros más. La justificación de algunos vaticinios de Mendeléiev como el descubrimiento de estos tres nuevos elementos y el surgimiento de un nuevo método de análisis químico, contribuyeron a resolver el enigma del Berilio, al precisarse su masa atómica y su posición en el sistema periódico enseguida del Litio.

Los contundentes éxitos implicaron la aceptación del Sistema Periódico de Mendeléiev por los grupos de científicos, al adoptarlo como una teoría de la periodicidad, orientadora de la búsqueda de nuevos elementos, de las correcciones a numerosas mediciones experimentales, de la creación de nuevos métodos de análisis químico y al cambio en concepciones equivocadas acerca de los elementos y compuestos químicos. Además, en la década del 90 del siglo XIX, en varios países, comenzó la publicación en los manuales de química del Sistema Periódico de Mendeléiev.

También , en la misma década, los trabajos de H. Becquerel (1852-1908), M. Curie (1867-1934) y P. Curie (1859-1906), acerca del descubrimiento de la radiactividad en algunos materiales naturales llamados radiactivos, provocó la búsqueda de nuevas sustancias, obteniendo un éxito insólito, al aislar los elementos radiactivos ya previstos por Mendeléiev: Radio, Polonio, Torio y, pocos años después, Actinio, por uno de los colaboradores de M. Curie, A. Debierne (1874-1949).

La naturaleza flexible y problemática de la investigación de Mendeléiev, así como su poder orientador del trabajo de químicos y físicos, también es manifiesta cuando a partir de 1878, en la sesión de la academia de Ciencias de París, se informa por los astrónomos Janssen (1824-1907) (francés) y Lockyer (18361920) (inglés), acerca del descubrimiento por detección espectroscópica del elemento Helio en las protuberancias del sol. Veinte años después, el físico inglés L. Rayleigh (1842-1919) y el químico W. Ramsay (1852-1916), luego de un minucioso trabajo para resolver el enigma de porqué el nitrógeno del aire era más pesado que el nitrógeno obtenido de otras fuentes identificaron al elemento Argón. Estos dos elementos químicos y otros descubiertos pocos años después: Kriptón, Neón, Xenón y Radón, tenían la característica de no participar 
en las interacciones químicas, a pesar de haber utilizado los científicos el método de Mendeléiev para el estudio de varias propiedades físico-químicas. Por lo tanto, hubo el problema de cuál posición tenían en la ley periódica, no había espacios libres para ellos y pareció presentarse el sistema periódico como una ley inexacta, situación que aprovecharon sus opositores para hacer sentir las voces de todos aquellos que dudaban de la periodicidad de los elementos químicos y no atribuían valor a la clasificación por inexacta e incompleta.

Sin embargo, en el año de 1900 D. Mendeléiev y W. Ransay, obtuvieron el triunfo de la flexibilidad del sistema, cuando en Berlín propusieron de manera conjunta una solución sencilla: disponer los gases inertes entre el grupo de los halógenos (VII, VIIA o 17) y el de los metales alcalinos (I, IA o 1), de manera que formen un grupo individual, denominado grupo cero (VIII, VIIIA o 18). Esta solución no perjudicaba en ningún punto la estructura del sistema periódi$\mathrm{co}$, por el contrario, lo hizo más armonioso y consumado.

No todos los problemas del sistema de Mendeléiev tuvieron solución, pero si la suficiente para ser un programa de "ciencia moderna» al estar produciendo problemas nuevos y resultados nuevos. No obstante, había problemas pendientes: el desorden de las masas atómicas de Argón y Potasio, Teluro e lodo, Cobalto y Níquel; la incierta posición del Hidrógeno; la posición de los elementos de las tierras raras, los cuales cada vez, aumentaban en número y creaban más confusión al presentarse unos como indistinguibles desde el punto de vista químico y con masas atómicas diferentes, otros, tenían masas atómicas similares y nada en común en sus propiedades. Esta última situación planteó un profundo interrogante al sistema de Mendeléiev: ¿Ubicar varios elementos en una misma casilla o quedar fuera del sistema?

La clasificación periódica de los elementos químicos para principios del siglo XX quedaba lista en su estructura fundamental. La última versión de Mendeléiev del sistema periódico, fue publicada en 1906 en la octava edición de sus "Fundamentos de Química", incluía el grupo cero y muchos nuevos espacios libres; no estaba definida la posición de los elementos denominados tierras raras. Las anomalías pendientes puntuaban la claridad acerca de la permanencia oscura de la razón última de la periodicidad misma (Chamizo 1991), la cual encontraría respuesta posteriormente en las investigaciones relacionadas con el núcleo atómico de $E$. Rutherford (1871-1937) y F. Soddy (1887-1956) sobre la desintegración radioactiva y los desarrollos de los modelos atómicos, en particular el mecanocuántico; los estudios relacionados con radiactividad fueron la causa, pocos años después, de la investigación científica de síntesis artificial de elementos químicos, liderada inicialmente por $\mathrm{E}$. Fermi (1901-1954) y E. Segré (1905 ) en Italia en el año de 1934. Las soluciones empezaron a vislumbrase con el progreso de estos programas de investigación, nuevos y afines del que nos ocupa. Éstos, acrecentaron el contenido de la ley periódica con supuestos básicos que eran ocultos a muchos investigadores químicos y físicos, dedicados a la medición exacta para probar teorías y a un falsacionismo ingenuo de oposición a la teoría atómica. El 10 de julio de 1905, Mendeléiev anotó en su diario :

"Por lo visto, el futuro no amenaza a la ley periódica con destruirla, lo único que le presagia es añadiduras y desarrollo". (Petriánov y Trífonov, 1981) 
El transcurrir del desarrollo científico hizo realidad este enunciado; la heurística negativa de la investigación de Mendeléiev, desde nuevos aportes de investigación, evolucionó a la metodología de identificación del núcleo atómico como elemento químico con el número atómico y un determinado número de neutrones, y de este modo, trascendió los supuestos básicos que estaban ocultos y ligados a la masa atómica, y la heurística positiva se ha enriquecido de nuevas relaciones de periodicidad de los niveles conceptuales molar, molecular y eléctrico.

\section{ETAPA DE INVESTIGACIÓN CIENTífICA DE E. RUTHERFORD Y F. SODdY}

La investigación de la escuela científica de E. Rutherford (1871-1937) y F. Soddy (1887-1956), científicos ingleses, adoptó un cambio creativo y revolucionario que transformó, cien años después, la hipótesis de la masa atómica de Prout (1785-1850), a pesar de las refutaciones en el fundamento de las teorías modernas sobre la estructura de los átomos. Este cambio, ya anticipado por W. Crooks (1832-1919) en 1888, cuando indicó que debería buscarse una nueva demarcación entre la física y la química y anticipaba que cada elemento químico tenía variedades que se distinguían por sus masas atómicas (Lakatos, 1983,74).

Rutherford y Soddy en 1902, propusieron la hipótesis según la cual, la desintegración radiactiva estaba acompañada de la transmutación de los elementos químicos y perfilaban la conjetura de que todos los elementos radiactivos estaban relacionados entre si de alguna manera. El concepto de elemento químico sufrió una profunda transformación. El significado de elemento quími- co como sustancia elemental contrastaba de modo contundente el significado lavoisieriano de elemento como sustancia simple manipulable y tratada por los métodos de separación y químicos tradicionales y daba razón a la fina intuición filosófica de Mendeléiev de una materia primaria.

Al abrirse paso la idea de la separación de los elementos químicos por métodos químicos nucleares, análisis químico de isótopos, en contra de que dos elementos de comportamiento químico similar deberían separarse por métodos físicos tradicionales, el concepto de elemento se modificó así como el concepto de átomo como partícula material indivisible e invariable se derrumbó y una tras otra verificaciones experimentales confirmaban núcleos atómicos y electrones, principalmente las vinculadas con los programas de investigación de E. Rutherford y de J. J. Thomson (18561940).

La investigación de Rutherford, dedicada a resolver la desintegración radioactiva, tuvo un éxito fantástico en 1908, al dilucidar experimentalmente la existencia del núcleo atómico de carga eléctrica positiva y lograr mediante diferentes verificaciones empíricas y diversos métodos de cálculo, demostrar que la carga eléctrica positiva del núcleo atómi$\mathrm{co}$, era aproximadamente igual a la mitad de la masa atómica para elementos livianos, mientras esta correlación no se confirmó para los elementos del centro y del final del sistema periódico.

El desarrollo progresivo de la investigación de Rutherford, estaba encaminado a aclarar la posición en el sistema periódico de Mendeléiev de los elementos de tierras raras, orientado por la hipótesis de los cambios en la naturaleza química del elemento cuando éste emitía 
radiación de alta frecuencia. La sistematización de varias teorías observacionales la llevan a cabo en 1913, F. Soddy y K. Fajans (1887-1975), al formular la ley del desplazamiento radioactivo en la desintegración alfa $(\alpha)$ : el elemento radioactivo se transforma en otro elemento, apartado éste del inicial, en dos posiciones a la izquierda en el sistema periódico, mientras en la desintegración beta $(\beta)$, se desplaza en una posición a la derecha.

En este mismo año, F. Soddy adoptaba un cuerpo teórico firme a la conjetura ya anunciada por Crooks, y propuso que los radioelementos con propiedades químicas similares se considerasen como variedades de un mismo elemento radioactivo, a las cuales denominó isótopos. Más tarde se aclararía que el fenómeno de la isotopía era inherente a todos los elementos químicos y no sólo a los radioactivos, era una característica de la estructura nuclear: los isótopos tienen igual número atómico y diferente número de neutrones, partícula esta última verificada en el año de 1932.

Las variedades isotópicas de los elementos químicos y la identificación del núcleo atómico con el número atómico y un determinado número de neutrones es un éxito de la investigación de Rutherford y una prueba muy fuerte para el sistema periódico de Mendeléiev; éste salió airoso, el núcleo firme fue precisado, en la búsqueda de los elementos el análisis químico llega hasta los isótopos, y el cinturón protector quedó a la espera de una solución completa a la posición de los elementos de tierras raras en la ley periódica.

También en 1913, el científico holandés A. van den Broek, físico teórico, propu so una conjetura intuitiva como criterio de ordenación. Apoyado en el progreso del programa de Rutherford, sugirió: "la carga nuclear del átomo de cualquier elemento es igual numéricamente al número atómico del elemento en el sistema periódico"

Esta hipótesis, fue sometida al poco tiempo, a verificación por el físico inglés Henry G. Moseley (1887-1915) de la universidad de Manchester. Al estudiar los espectro atómicos de rayos $\mathrm{x}$ de varios metales de transición, demostró que la longitud de onda característica de los rayos $x$ emitidos, varía con regularidad al pasar de un elemento a su vecino inmediato en una fila del sistema periódico. El número atómico se podía determinar al establecer una relación entre éste y la longitud de onda de la radiación de rayos $x$ emitida. El valor del número atómico resultó ser idéntico a la carga eléctrica positiva del núcleo atómico. La relación de Mosely, detallada a continuación, muestra el correlato empírico ( $\lambda$ de rayos $x$ ) del número atómico y este adquiere la categoría de observable mediante la espectroscopía de rayos $-\mathrm{x}$ :

$$
1 / \lambda=(Z-1)^{2} R(3 / 4)
$$

$\mathbf{Z}$ número atómico; $\lambda$ longitud de onda de los rayos- $x$ emitidos; $\mathbf{R}$ conste de Rydberg

Una función matemática de raíces empíricas establece que el inverso de la longitud de onda depende estrictamente y de modo directamente proporcional, del número de protones en el núcleo atómico. Este fenómeno no está relacionado con los electrones externos de los átomos sino con aquellos más cercanos al núcleo atómico. Este nuevo conocimiento aportó en una primera instancia modificar la organización pe- 
riódica de los elementos químicos de Mendeléiev en términos de las variables masa atómica y valencia por las variables número atómico y valencia.

Lo que fuera una hipótesis intuitiva, devino en uno de los más geniales y transcendentales desarrollos superiores del sistema periódico. Este obtuvo una fundamentación química y física en un nivel nuevo y más alto del conocimiento científico. El enunciado central de la ley periódica se transformó en: "Las propiedades de los elementos y sus compuestos son función periódica de la magnitud de las cargas nucleares de sus átomos" (A.v.d. Broeck, 1913). La heurística positiva de la investigación de Mendeléiev fue sustituida por una nueva hipótesis atómica de mayor contenido científico y a la vez relacionadas de modo dialéctico, dado que el núcleo se identifica por el número de protones $(Z)$ y el total de partículas nucleares, protones y neutrones o número másico (A).

La base de la ley periódica no eran ya las magnitudes de las masas atómicas que incrementaban en una fila al pasar de un elemento a otro sin subordinarse a una ley explícita, sino los valores enteros de la carga eléctrica positiva de los núcleos atómicos o número atómico, los cuales obedecían a la ley de Moseley y variaban una unidad exactamente al pasar de un elemento a otro en la fila. Una casilla de la tabla periódi$c a$, identificada por Z, representa al elemento químico y sus formas isotópicas. A la noción de masa atómica le sucede como variable organizadora el número atómico, un significado más abstracto desde lo fenomenológico. Este número adquiere un doble significado, por un lado, tiene naturaleza ordinal al determinar el orden de las sustancias elementales en las filas, y por otro, es de naturaleza cardinal al permitir contar el número de protones en el núcleo atómi- co y en consecuencia el número de electrones para las partículas eléctricamente neutras y en sus formas iónicas.

El sistema periódico de Mendeléiev, se transformó a si mismo en un nuevo sistema periódico, con un núcleo firme enriquecido de contenido al avanzar los métodos de separación y análisis químico hasta las formas isotópicas de los elementos y su identificación con el número atómico, basado en una nueva hipótesis atómica ligada en su origen a la solución de las anticipaciones de Prout y de Crooks acerca de las masas atómicas, con toda la riqueza de la heurística positiva del programa de Mendeléiev y con un crecimiento de ésta, al hallar solución al problema no resuelto de las posiciones de argón y potasio, cobalto y níquel, telurio y yodo; además, se pudo afirmar con exactitud que el sistema natural empezaba con el hidrógeno $(Z=1)$ y terminaba con el uranio $(Z=92)$. Los científicos podían afirmar con claridad cuales elementos vaticinados por Mendeléiev no habían sido descubiertos para la época: tecnecio $(Z=43)$, renio $(Z=45)$, hafnio $(Z=72)$ y prometio $(Z=71)$; permanecía el enigma de los elementos con número atómico 85,87 y 91, también predichos por Mendeléiev. Varios de estos elementos serían encontrados quince años después mediante refinadas, prolongadas y minuciosas búsquedas experimentales.

Los elementos con número atómico 43 , 61 y 85 , no fue posible evidenciarlos en la búsqueda experimental, a pesar de que los científicos disponían de un poderoso método que les indicaba inequívocamente, el camino de indagación, al tener conocimiento por anticipado de varias propiedades químicas del elemento desconocido. Su solución estuvo involucrada en una nueva etapa de investigación, la síntesis artificial de elementos químicos o nucleosíntesis 
artificial, que para mediados de la década del 30 del siglo XX ya era un programa de investigación en marcha.

De este modo "La búsqueda de los elementos químicos" es un programa de investigación a largo plazo que incluye subprogramas y épocas diversas, conceptualizaciones y reconceptualizaciones, nuevos modos de pensamiento acerca del mundo y nuevos modelos nucleares y moleculares, en particular acerca de los elementos químicos y las sustancias químicas, y de modo contundente nuevos métodos de separación y análisis químico cada vez más refinados y de mayor contenido racional. Las sustancias simples de Lavoisier con sus respectivas correcciones, varios de los cuerpos simples de hoy, ha evolucionado a través de los métodos químicos y de varios modelos a una forma no tangible ni manipulable de modo directo e implicada en un alto nivel de abstracción.

La química de la época actual diferencia de modo claro las sustancias simples y los elementos químicos o sustancias básicas o primarias, nombre dado por Paneth (1962) para establecer los límites entre sustancia simple y elemento químico y su diferencia del significado de átomo o molécula mononuclear. Una hipótesis atómica, en un momento la masa atómica, en otro el número atómico y en ambos casos ligada de modo dialéctico a la valencia química se constituye en la heurística positiva cada vez con mayor contenido empírico, conceptual y predictivo, enriquece la comprensión y la inferencia acerca de los comportamientos de las sustancias y la síntesis de nuevas sustancias simples y compuestas y de nuevos elementos químicos.

\section{Consideraciones finales}

El gran programa de investigación, la búsqueda de los elementos toma forma científica mediante una ruta operacional con cada vez mayor contenido racional. El sistema periódico bidimensional propuesto por Mendeléiev tiene no sólo la capacidad de sistematizar lo que sus predecesores y contemporáneos habían elaborado acerca de la ordenación de los elementos, sino que tiene de modo principal la gran capacidad de predecir la búsqueda de nuevos elementos al anticipar características de éstos y de varios de sus compuestos. De este modo, el programa de Mendeléiev potencia el núcleo firme al guiar la dilucidación de nuevas metodologías analíticas como las espectros-cópicas y nucleares para el encuentro e identificación de nuevos elementos y de los ya conocidos tiempos atrás.

La búsqueda de los elementos en continua evolución, marcada del signo revolución, vive en la época moderna de pensamientos verificados y de experiencias de gran precisión que han dado demostraciones de validez y mayor poder de explicación. La sistemática de las sustancias básicas o elementales ha trasegado las técnicas de separación e identificación en los niveles físico, químico y nuclear con una mayor extensión de racionalidad en cada nivel, con una mayor profundidad científica de la experiencia y con una gran sistemática de organización del saber en la tabla periódica.

La pluralidad de las experiencias físicas, químicas y nucleares implica la diferenciación y las relaciones entre elementos, sustancias simples y compuestas, explicitadas en el sistema periódico mediante relaciones abstractas en términos de la organización cruzada de información química y física, en grados jerarquizados de abstracción. Esta organización permite potenciar los procesos de inferencia acerca de las sustancias y sus propiedades. 
El reconocimiento de la historia y los aspectos epistemológicos del progreso conceptual del sistema periódico implica reconocer el progreso conceptual de las técnicas de análisis, de la noción de elemento químico y de la ordenación cruzada en términos de variables cuyos significados se multiplican con el progreso científico. Este reconocimiento permite comprender como un fenomenología esencialmente química, como es la de Mendeléiev, es profundizada por una noción fenomenológica más abstracta, el número atómico, que asume la ordenación de los elementos cruzado con la valencia.

Este reconocimiento histórico-epistemológico de la búsqueda de los elementos, implica consideraciones didácticas en cuanto que la tabla periódica como objeto de enseñanza para un aprendizaje significativo, requiere la comprensión de su historia y de su progreso conceptual en términos de la organización primero lineal y luego cruzada de información química y física en grados jerarquizados de abstracción. Esto conlleva la organización de situaciones didácticas según características de grupos de alumnos en un dado grado escolar. Para los profesores es una recontextualización de su saber científico y didáctico para orientar la apropiación de que saben los alumnos y como lo saben, que conocimientos organizados presentar y que actividades de aula planear.

La apropiación por parte de grupos de docentes de los procesos históricos y epistemológicos de la creación científica, en particular del sistema periódico de los elementos químicos, contribuye a superar lo que la investigación adelantada y la experiencia docente manifiestan acerca de un aprendizaje de la tabla periódica como un formato acabado, cuyo único objetivo es leer datos aislados de casos particulares de elementos químicos. Leer lo básico, está asociado a la no comprensión de los conceptos implicados en los datos, traducidos estos a números, que en la mayoría de los casos son entes sin significado, ya que la unidades correspondientes se omiten, así como el significado de la propiedad y la clase de sustancia a la cual están vinculados.

Igualmente contribuiría a reconocer en primer lugar el arte de clasificar y describir en química, según una amplia diversidad de criterios concretos y abstractos, y en segundo lugar a detectar los vínculos de periodicidad de los elementos y las diferencias conceptuales entre elemento químico, sustancia simple y sustancia compuesta, entre sus comportamientos químicos y físicos y sus profundas conexiones con los modelos nuclear y molecular.

Finalmente, varios químicos preocupados por la pedagogía de la tabla periódica como por ejemplo, Fernelius (1986), plantea que al usar la ley periódica, ya sea para organizar su conocimiento o para predecir acerca de lo desconocido, debe tenerse primero la comprensión del sistema periódico, antes de realizar conjeturas razonables sobre algo desconocido; considera que el conocimiento de la tabla periódica ayuda a disminuir la entropía del conocimiento. Comprender el sistema periódico, es comprender su progreso histórico y episte-mológico, con el fin de desarrollar una pedagogía mas adecuada de este sistema.

\section{REFERENCIAS BIBLIOGRÁFICAS}

ASIMOV, Isaac. 1983. La Búsqueda de los Elementos, Barcelona: Plaza \& Janes, S. A.

CRUZ, CHAMIZO, GARRITZ. 1986. Estructura Atómica Un Enfoque Químico, México: Fondo Educativo Interamericano S. A. 
FERNELIUS, W., Conrad. 1986. Some Reflections on the Periodic Table and its Use, Journal of Chemical Education, 63, 3, 263-266

GORIN, George. 1996. Mendeleev y Moseley, The Principal Discoverers of the Periodic Law Journal of Chemical Education, 73, 6, 490-493

LAKATOS, Imre, 1978, La Metodología de los Programas de Investigación Científica", Madrid: Alianza Editorial.

LAVOISIER, Antoine, L.1982. Tratado Elemental de Química, Madrid: Ediciones Alfaguara.

LEFORT, Marc. 1989. Los Últimos Elementos de la Tabla de Mendeleev, Mundo Científico, 9, 95 966-973.

MENDELEEV, Dimitri. 1905. Principles of Chemistry I, Vol. 1, London: Longmans, en Scerri, R. Eric, 2000, Realism, Reduction, and the "Intermediate Position", in Bhushan, N.,

MOORE, F. J. 1953. Historia de la Química, Madrid: Salvat Editores.

PANETH, Fritz, A., 1962, The Epistemological Status of the Chemical Concept of Element (I), The Brithist Journal for the Philosophy of Science, Volume XIII, No. 49, pag. 1-14.

PANETH, Fritz, A., 1962, The Epistemological
Status of the Chemical Concept of Element (II), The Brithist Journal for the Philosophy of Science, Volume XIII, No. 49.

PETRIÁNOV, I. V., Trífonov, D. N. 1981. La Magna Ley, Moscú: Editorial Mir, (Traducción del ruso Clara Shteinberg).

SABINO, A., Carlos. 1999. Por los caminos de la Ciencia, Santa Fé de Bogotá: Editorial Panamericana.

ROUNDY, Willard, H., Jr. 1989. What Is an Element?. Journal of Chemical Education, 66, 9, 729-730.

SCERRI, R. Eric. 1998. Evolución del Sistema Periódico, Investigación y Ciencia, Noviembre, 54-59.

SCERRI, R. Eric. 2000. Realism, Reduction, and the "Intermediate Position", in Bhushan, N., Rosenfeld, S., Of Minds and Molecules, New Philosophical Perspectives on Chemistry, New York: Oxford University Press, Inc.

SEABORG, T. Glen. 1985. The Transuranium Elements, Journal of Chemical Education, 62, $6,463-467$

STRATHERN, Paul, 2000, El Sueño de Mendeléiev, de la Alquimia a la Química, Madrid: Editorial Siglo Veintiuno. 